420 8o6. 26 septembre roro. Karl Burkheiser.

426 r25. I6 février rgr . Mason (The suJphate... elc.).

427 o65. I8 mai I9ro. Soc. industr. de produits chimiques.

14 046 . 24 juin rgro. Soc. industr. de produits chimiques.

15307.29 mars 9 r. Soc. industr. de produits chimiques.

429 708. п3 mai r91. Karl Burkheiser.

43 г $2 / 4$. I7 juin rgr. Walther Feld.

13259o. 17 juin rg⿵. Walther Feld.

434 232. 5 septembre y gro. Braunschild el Chapiro.

1/4662. is novembre igro. Braunschild ef Chapiro.

434 673. 2 décembre rgro. Gouthière et $\mathrm{C}^{\mathrm{ie}}$, et Dueancel.

5 449. 2 mai rgr. Gouthière ot $\mathrm{C}^{\mathrm{ie}}$, et Ducancel.

436768.28 octobre rgir. Gewerkschaft des Stcink. Iothr.

$437257 . \quad 7$ décembre igx. Fabry.

$43_{9} 365$. 4 avril rgr. Gouthière ol $\mathrm{C}^{\mathrm{ie}}$, et Ducancel.

4 $4033 \mathrm{r}$. er $^{\text {mai }}$ I $9 \mathrm{~s}$. Burgevin.

44 1573. 20 mars igr2. Karl Burkheiser.

445 т62. I9 juin r9 2. Frederic William Burstall.

445556.28 juin r9 9 . Karl Burkhoiser.

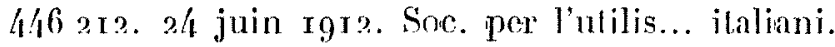

14. Azote. - Transformations de produits azolés en d'autres produits azotés (non classés déjà aux séries $5^{\mathrm{e}}$ et $13^{\circ}$ de l'azote).

41/682. 9 avril r9ro. Freth ol Edwin Cocksedge.

r35 го. 5 décembre rgıo. Freeh el Edwin Cockselge.

4 í9 $78 \% 27$ août 1910. Karl Kaiser.

420258.7 septembre s 910 . Erust. IIenss.

42 85 г. 26 octobre г9 ro. Edgar Arthur Asheroft.

426307.2 f fevier rga r. Flektrochemische Werke.

428 or 4 . I juin rgro. Braunschild el Chapiro:

429708 . 18 mai r9ı. Karl Burkheiser.

430 93 г. r3 juin rgr . Freeth et Cocksedge.

43r 058. I4 juin igr. Freeth et Cocksedge

43r o88. 3 r août r 9ro. Gouthière et Ci, el Ducancel.

433 3o6. 3 août rgr I. Alby. U. C. F. Ld.

135397 . 8 octobre r9 1... Karl Kaiscr.

435476 . 28 seplembre ig r. The New Jersey Zine C.

436 185. 16.janvier ig1 Guignard el Watrigant.

I5 o80. 1o fevier rgir. Guignand el Watrigant.

436768.28 oclobre 191 . Gerverkschaft des St. Iolhr.

437 I68. to février ig 1 r. Sociélé générale des nilrures.

438 э48. 9 mars rgr. Société générade des nilrures.

438487. 2 décembre 1911. Chemische Fabrik ron Jeyten

$4389_{965}$. т3 janvier ig12. ITenrich sulzer.

hit2 og2. 20 mars rgre. Lemaire el de Geyter.

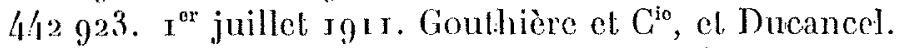

r6 297. $\mathrm{r} 7$ octobre rger. Goulhiere et $\mathrm{C}^{\mathrm{io}}$, ol Durancel.

4h5 857. 15 septembre ig1r. A de Montlatr.

4h7 ro6. ro août rger. Emil Collet.

4473 : r. 7 août igr2. Edgar Arthur Asheroft.

Cette nomenclature donne une idée frappante de l'incomparable activité que les chercheurs déploient pour atteindro la solution de ce problème si important posé dès les origrines de la scienes chimique : l'industrialisalion de l'azote. Fn remarquant que celle longue liste de brevets ne comprend que ceux délivrés ces deux dernières annécs, el en France seulement, tandis qu'à l'étranger, notamment en Allemagne, dans les Pays Scandinaves, en Angleterre et aux Etats-Unis, benucoup d'autres brevets ont été pris qui n'ont pas ćté de. mandés en France, on juge quelle doit être la somme considérable des Iravaux s'accumulant.
La semence jetée par celle foule de travailleurs contient assurément de l'ivraie, c'est-à-dirc un certain nombre de conceplions pratiquement irréalisables, mais aussi des idées justes et des découvertes de valeur - les bons grains - et il ne peut y avoir de doute : la moisson sera belle. Nous avons la conviction que te champ de la houille blanche fera pour sa parl une abondante récolte.

E.-F. Côte.

\section{TRAMWAYS ÉLECTRIQUES}

\section{Voitures à plancher surbaissé et sans marchepied des " New-York-Railways"}

Une des Compagnies qui desservent New-York, la NewYork Railways Company, a, cetle année, mis en service une voiture sans marchepied qui, ainsi qu'on s'cn convaincra facilement par l'aspect de la figure $I$, constitue l'innovation la plus originale el la plus hardie qui ait été depuis long-

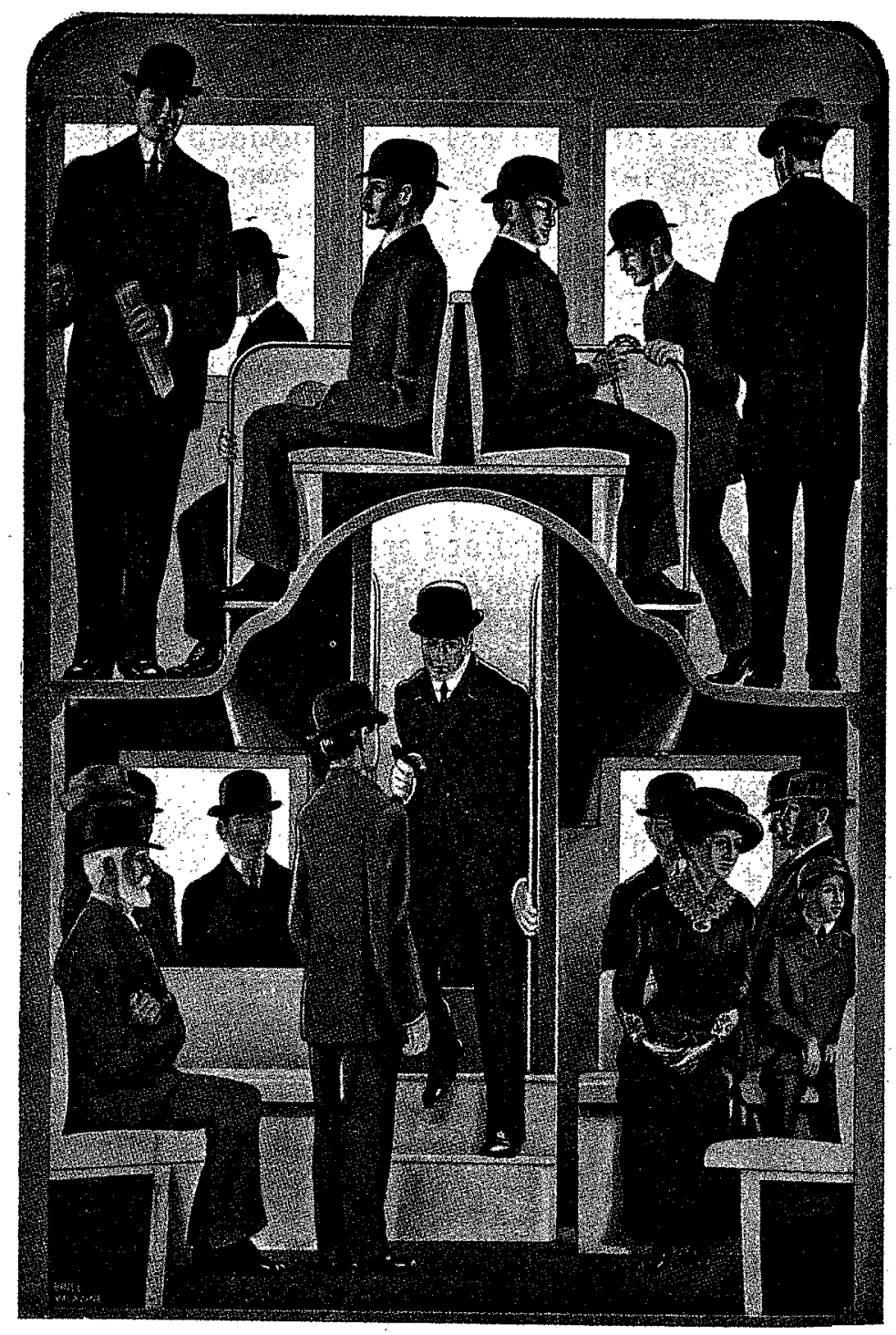

FIG. I. - COUPE TRANSYERSALE DE LA VOITURE.

temps réalisée dans la construction des voitures. On sait ì quels accidents de toute sorte donnent lieu les marchepieds; le plus souvent peu ćludićs, des voitures de laamways, et quelle gêne et quels relards ils occasioment, trop souvent ì la monlée, ou à la descenle. Les ingénieurs de New-York onl appliqué au mal le remède le plus radical qui soit a ils ont purement et simplement supprimé le marchepied, of 


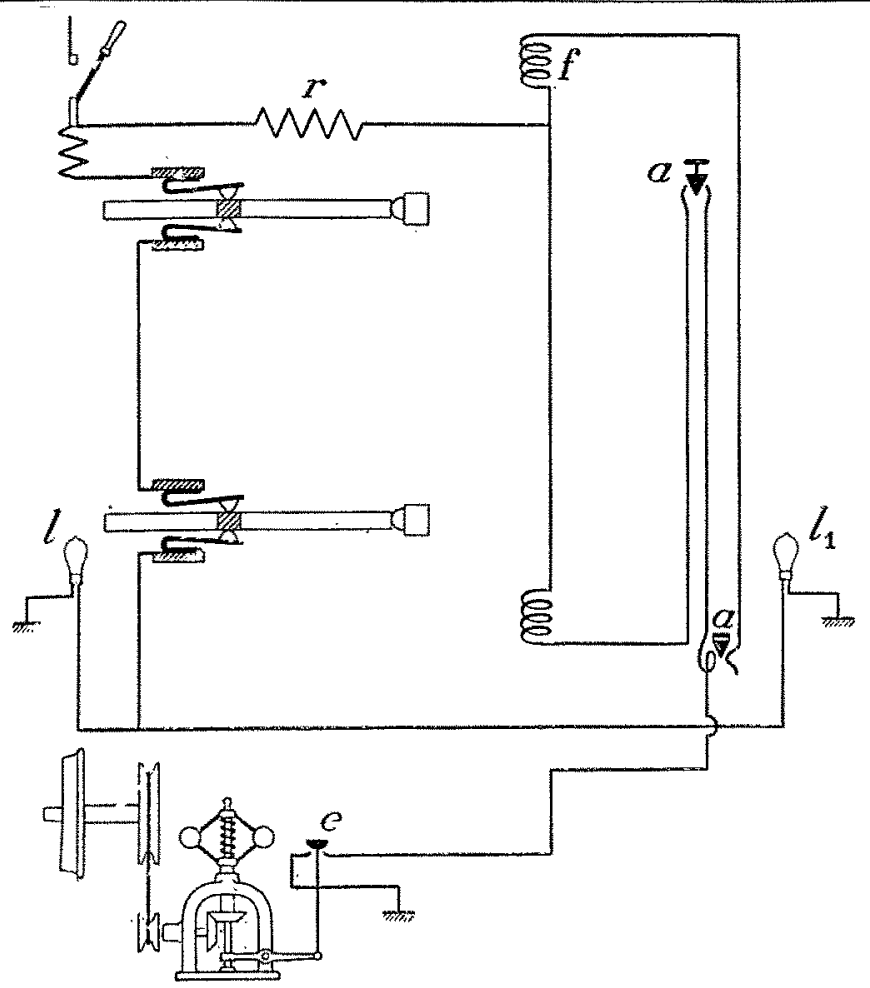

FG. 3. - dispositif de VERROUILLAge des PORTES PENDANT LA MARCHE e, contact fermé seúlement quand la voiture est au repos. - a, contact permettant l'ouverture et.$l$ a fermeture de la porte. $-i$ et $j_{1}$, lampessignal placées dans lacabine du wattman. $-r$, résistance. à l'entréc, et font tomber dans la caisse enregistrense le prix de la place. La plateforme médiane sert seulement à l'entrée ot à la sortie, el les voyageurs ne sont pas admis à y sta. lionner autrement.

Les dispositifs d'ouverture automatique de la porte et d'immobilisalion de celte dernière, tant que la porte n'est pas fermée, sont également fort intéressants of en grante partic inédits.

On s'est proposé de réalisere les condilions suivantes:

$1^{\circ}$ Les portes ne peuvent s'ouvirir tant que la voiture est on marche ;

$z^{\circ}$ La voiture ne peut démarrer tant que les portes sont ouvertes.

Pour obtenir le premier de ces résultats, un régulateur ì boules, commandé par les rones de la voiture, maintient ì l'ouverture, tant que les roues toument, un interruptenr' qui a pour cffet de couper te circuit commandant l'ouverture: de la porte, circuit qui est fermé par un bonton ponssoir mis it la disposition du conductem.

Pour réaliser le deuxieme desideratum, of immobiliser la voiture tant que lis portes no sont pas fermées, celles-ci, par un système de contacls mélalliques glissants, viennent établir on conper les circuits de commando dis contacteurs des moteurs. Les schémats des monlages sont représentés par les figures $3 \mathrm{el} / 4$.

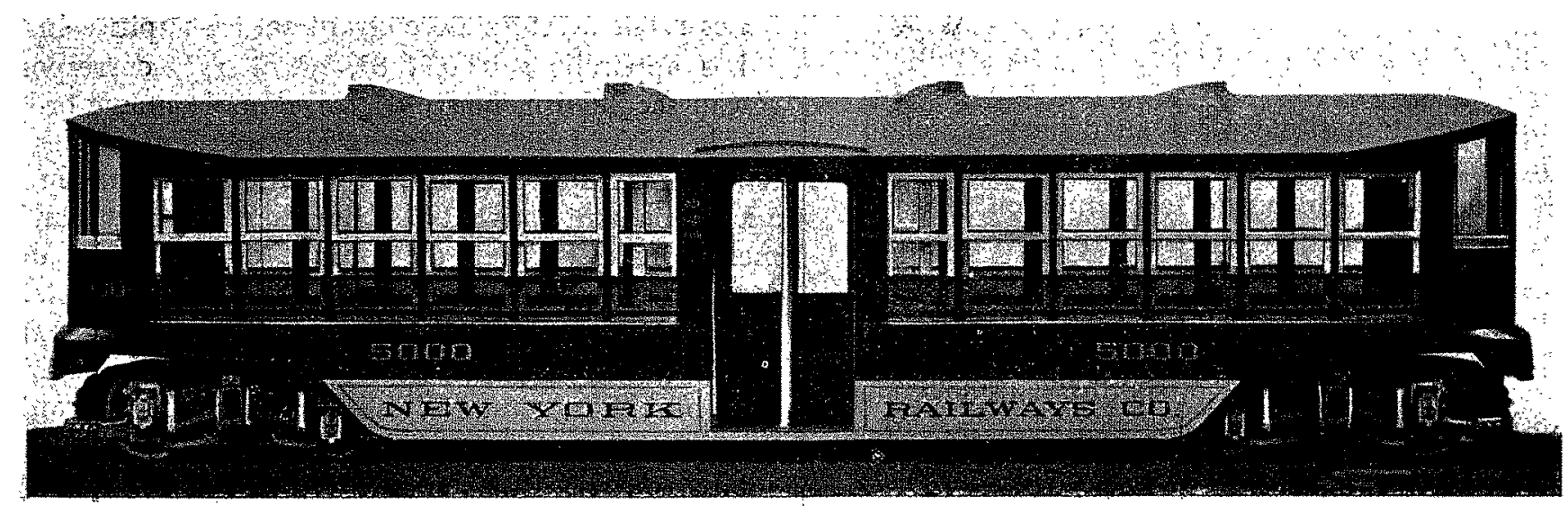

FIG. 2. - VUE LONGITUdinale DE LA vOTTURE SANS MARCIEPIED DES ( NEW-YORK RALWAYS ),

sont anvivés à construire une voilure ì plancher surbaissó dont le niveau n'est supéricur que do 25 centimètres à celiri de la voie.

L'originalité de la solulion adoptée consiste dans l'effacement des bogies extrêmes sous le creux des banquelles semicirculaires placées au deux bouts de la caisśe, de plancher lui-mème no próscntant, el c'est la le trail te plus caractérislique, ancun décrochement diun boul a lautre mais bunt senjement en pente légère des extrénilés vers la partie médiane qui sert ì lientrée di à la sorlie.

Les figures $\mathrm{r}$ on 2 montrent la vollure en vue latérale et en coupe transvepsale; clles font comprendre mixux que toute description la disposition de l'ensemble.

la longueur tóbale est de $12^{\mathrm{m}} / \mathrm{ho}$ et la largeur de $2^{\mathrm{m} 5} 5 \mathrm{r}$. I'érartement des axe's des trucks est de $8^{\text {m. }}$. Ces trucks sont du type it un senl moteur ; its comportent une paire de rones motrices de $o^{\mathrm{m}} 701$ de diamètre, et une paire de roues porteuses de o $\mathrm{m}^{\mathrm{m}} / \mathrm{s}$.?

La pereption du prix des places se fail à l'entrée, systeme rendu possible par l'uniformitó lu tarif (5 cents). les voyageurs défilent pour entrer devant le receveur assis au fond de la plateforme médiane dans la partic opposéc

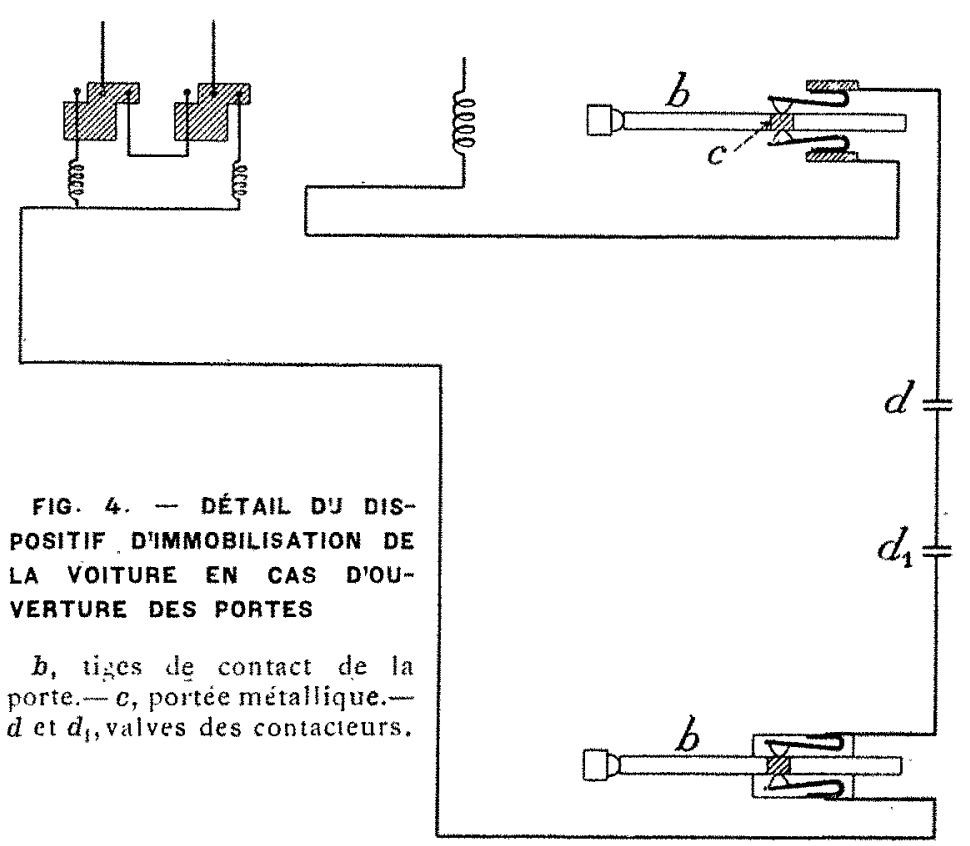

La voiture est munic da nouveau dispositif de command: a unités multiples, type HI., qui est lataptation aux voituran 
de tramways, avec une réduction de poids et d'encombrement, du systeme bien connu appliqué aux chemins de for de grandes lignes el aux métropolitains, et dont le principe (fermeture successive de contacteurs à l'aide de servomoteurs gouvernés par relais), est trop connu pour avoir besoin d'être décrit ì nouveau.

Ultérieurement à l'apparition de ces premières voitures qui étaient sans impériale, la Compaguie a songé à lirer parti de labaissement de la caisse, que permet la solution du plancher surbaissé, on agençant un nouveau modèle à impériale qui peut contenir en tout $77^{x}$ voyageurs.

P. Bourgutonon, Ingénieur-Electricien.

\section{INTERRUPTEURS AUTOMATIQUES}

\section{Réglage des interrupteurs automatíques placés en série sur une canalisation (4)}

Si, dans un réscau de distribulion de courant, nous sûivons le tracé des canalisations depuis l'origine, c'est-a-dire depuis l'usine centrale jusqu'uux points d'utilisation de l'énergio électrique, nous constatons, en général, la présence des apparcils de sécurilé suivants :

$1^{\circ}$ Interrupleur aulomatique placé aux bornes des alternateurs de la Centrale;

$2^{\circ}$ Interrupleur aulomatique placé sur un grompe de feeders ;

$3^{\circ}$ Interrupteur automatique placé au départ sur chaque feeder, alimentant tes sous-stalions de transformation ou les postes de transformalion ou de scctionnement;

$4^{\circ}$ Interrupteur sur chaque fecder, à ]'arrivée dans les sous-stalions, postes de thansformation ou de sectionnement;

$5^{\circ}$ Interrupteur automatique sur chaque nouveau feeder ou distributeur sontant d'un poste de sectionnement. S'il s'agit de distributeurs bouclés, d'autres interrupleurs automaliques sont souvent intercalés dans ces distributeurs pour la marche en parallèle;

$6^{\circ}$ Interrupteur automalique avant l'cntrée de chaque convertisseur rotalif ou transformateur statique dans les sousstations on les postes de transformation;

$7^{\circ}$ Interrupteur automalique placé aux bornes de chaque convertisseur rolatif (côté couranl continu) ou de chaque transformaleur statique (cólé basse tension);

$8^{\circ}$ Interrupteur automatique sur chaque feeder sccondaire; $9^{\circ}$ Coupc-circuit ou interruptcur automatique ì retour de courant à l'arrivée de chacun de ces feeders sur le réseau à basse tension;

$10^{\circ}$ Interrupleur automalique ou simple permettant la marche en parallèle ou le sectionnement du réseau en différents tronçons ;

$x^{\circ}{ }^{\circ}$ Coupe-circuit ou interrupteur automatique placé au départ de chaque dérivation.

On comprend facilement qu'avec un nombre aussi grand d'appareils en sćrie, la queslion du réglage do ces appareils soit assez difficile sinon impossible à résoudre d'une façon pratiquement saticfaisante. Aussi il faut rechercher le moyen de réduire le nombre des appareils automatiques au plus strict indispensable, surtout lorsque les courants agissant dans chaque interrupteur en séric sont du mème ordre.

(1) Résunḱ du rapport présenté par M. Nrcolint au Comité technique du "Syndicat protessionnel des Usines d'électricité "fublié in-extenso par La Revue Electrique (g aout Igra).
Les interrupteurs nommés sous $1^{\circ}, 2^{\circ}, 3^{\circ}$ et $5^{\circ}$ sont, en général, des interrupteurs automatiques à maxima, à déclanchement retardé, munis de relais spéciaux permettant ce déclànchement à temps.

Pour les interrupteurs destinés aux alternateurs de la Centrale, il y a des constructeurs qui préconisent, outre les interrupteurs à maxima, des interrupteurs à retour de courant, mais, à notre avis, cefte disposition ne peut présenter aucune utilité. Elle peut même, an contraire, donner lieu à des déclanchement intempestifs dans le cas d'une mise en parallèle défectueuse.

Par contre, certains exploitants conseillent même la suppression des interrupteurs automatiques à maxima sur les allernateurs.

Les interrupteurs sous $4^{\circ}$ sont le plus souvent des inter. rupteurs automatiques à retour de courant.

S'il s'agit d'un poste de sectionnement, à l'arrivée du feeder dans ce poste, on peut admettre de simples couteaux de sectionnement ou tout au plus un interrupteur simple à huile, se bomant i melte des interrupteurs automatiques à maxima et à temps sur chaque nouveau feeder sortant du posle (interrupteur sous $5^{\circ}$ ).

Les appareils sous $6^{\circ}$ so composent d'interrupteurs automaliques à maxima.

Ies relais à maxima peuvent être à déclanchement instantané ou à temps.

Les relais à retour de courant sont à rupture instantanée.

Les appareils sous $7^{\circ}, 8^{\circ}, 9^{\circ}$ et $\mathrm{I}^{\circ}$, correspondant au réseau secondairc, peuvent être, dans le même ordre d'idées que les précédents, destinés à la haute tension des interrupteurs a maxima ou à retour de courant, suivant le cas. Coux à maxima sont souvent à déclanchement instantané, nolamment lorsqu'il 's'agit de courant continu; ou de simple coupe-circuit, lorsqu'il s'agit de petites derivations.

Le rôle à remplir par ces interrupteurs automaliques est do protéger les apparcils ou les cables a l'origine desquels ils sont branchés, c'est-à-dire interrompre le courant aussitôt que celui-ci peut devenir dangereux.

S'ils sont réglés à temps, ils doivent couper pour des valeurs de courant en fonclion du débit normal des différentes canalisations et suivant des temps variables, selon une courbe dont certains points sont déterminés suivant les données ré latives aux installations à protéger ; les autres points résultent de la caracléristique mécanique des relais employés.

Les trois points qui constituent la base du réglage de ces interrupteurs sont les suivants.

$I^{\circ}$ Surcharge durable en pour roo à admettre comme limite maxima sur les installations à protéger et temps de déclanchement relatif sous la charge résultante;

$2^{\circ}$ Valeur maxima du courant de court-cicruit correspor dant à l'interrupteur considéré et temps relatif de déclanchement de cet interrupteur sous l'action de ce court-circuit;

$3^{\circ}$ Si plusieurs interrupteurs se trouvent en série sùr une canalisation, une autre condition essentielle à remplin est que les relajs de ces interrupteurs doivent être conçus de lacon à conserver leur action sélective dans tous les cas de surcharges admises dans les différénts tronçons de canalisation, y compris le cas d'un court-circuit franc pouvant avoir lieu dans un point quelconque de cette canalisalion.

Pour ce qui concerne le róglage des interrupteurs en sérié, pour des surcharges durables de l'ordre de 25 a 50 pour too de la charge normale, le problème est assez facile à résoudire: surtout-si les interrupteurs cn série correspondent à des tron- 